\title{
ESTRADIOL E PROSTAGLANDINA NA CONCEPÇÃO DE VACAS NELORE SINCRONIZADAS PARA IA EM TEMPO FIXO
}

\author{
OESTRADIOL AND PROSTAGLANDIN ON CONCEPTION RATE OF FIXED-TIME \\ INSEMINATED NELLORE COWS
}

\author{
Melo, W.O. ${ }^{1}$, Souza, J.A.T. ${ }^{2}$, Elias, A.K.S. ${ }^{1}$, Rocha, I.J. ${ }^{1}$, Conceição, E.J. ${ }^{1}$, Martínez, J.J.M. ${ }^{3}$, \\ Valarelli, R. ${ }^{3}$ e Torres-Júnior, J.R.S. ${ }^{4}$ \\ 'Universidade Federal Rural da Amazônia. Instituto da Saúde e Produção Animal. Belém. Pará. Brasil. \\ ${ }^{2}$ Universidade Federal do Piauí. Teresina-PI. Brasil. \\ ${ }^{3}$ Laboratórios Pfizer Saúde Animal. São Paulo-SP. Brasil. \\ ${ }^{4}$ Universidade Federal do Maranhão. Centro de Ciências Agrárias e Ambientais. Chapadinha. Maranhão. \\ Brasil. ttorresjunior@yahoo.com.br
}

\author{
PalaVRas chave adicionais \\ Bovinos. IATF. Sincronização. Ovulação.
}

\section{RESUMO}

Objetivou-se avaliar a taxa de concepção em vacas Nelore, sincronizadas para inseminação artificial em tempo fixo. Foi testada a eficiência do cipionato (CE) ou benzoato de estradiol (BE) como indutor atresia e emergência de nova onda folicular no inicio do tratamento dia 0 (D0) com dispositivo de progesterona $\left(\mathrm{CIDR}^{\circledR}\right)$. Também se verificou a aplicação de prostaglandina no momento da remoção do dispositivo dia 9 (D9; protocolo de três manejos) ou $48 \mathrm{~h}$ antes dia 7 (D7; protocolo de quatro manejos). Adotou-se delineamento experimental fatorial 2x2 (CE vs. BE e PGFD7 vs. PGFD9). Não houve diferença entre tratamentos $(p>0,05)$, sendo possível utilizar o protocolo com apenas três manejos.

\section{SUMMARY}

The objective was to evaluate the pregnancy rate of Nellore cows submitted to estrous synchronization for fixed-time artificial insemination. The efficiency of the estradiol cypionate (CE) and benzoate (BE) to induce atresia and new follicular emergence wave in the beginning (day 0 ; D0) of progesterone device $\left(\mathrm{CIDR}^{\circledR}\right)$ treatment was tested. The administration of prostaglandin at CIDR device withdrawal (day 9; D9, three handlings protocol) or $48 \mathrm{~h}$ before it (day $7 ; \mathrm{D} 7$, four handlings protocol) was also verified. A $2 \times 2$ factorial design

Recibido: 20-2-09. Aceptado: 5-3-09.

\author{
AdDitionAl KEYWORDS \\ Cattle. FTAI. Synchronization. Ovulation.
}

was established (CE vs. EBE and PGFD7 vs. PGFD9). No differences were showed between treatments $(p<0.05)$, being possible to employ the three handlings protocol.

\section{INTRODUÇÃO}

A tecnologia de inseminação artificial, associada a um manejo adequado do rebanho, têm sido empregada por técnicos e produtores, visando aumentar a qualidade e a quantidade de bezerros genética e fenotipicamente superiores.

A baixa taxa de serviço em bovinos inseminados artificialmente, é decorrente de falhas na detecção do cio, principalmente em rebanhos Bos indicus (Baruselli et al., 2004), que apresentam alto percentual de cios de curta duração ou noturnos (Galina et al., 1996).

Progestágenos exógenos associados a ésteres de estradiol têm sido utilizados para ocasionar, via supressão dos pulsos de FSH e LH, a atresia da onda folicular presente, seguido da emergência de uma nova onda de crescimento folicular após o restabelecimento dos pulsos de FSH (Bó et 
al., 1993; 1995). Assim, pode-se prover o controle da dinâmica de crescimento folicular e a sincronização da ovulação, permitindo a inseminação artificial em momento pré-determinado sem a necessidade de observação de cios (Yavas e Walton, 2000).

Juntamente aos tratamentos à base de progestágenos é preconizada a aplicação de prostaglandina $\mathrm{F}_{2} \alpha\left(\mathrm{PGF}_{2} \alpha\right)$ para indução da luteólise e queda dos níveis endógenos de progesterona (Dieleman et al., 1986), que eventualmente pode potencializar o crescimento final do folículo dominante e a ovulação (Barros e Ereno, 2004).

Nesta perspectiva objetivou-se, verificar a taxa de concepção de vacas Nelore, sincronizadas para IATF com uso de diferentes ésteres de estradiol no inicio do tratamento (D0) com dispositivo intravaginal de progesterona e diferentes momentos de aplicação de $\mathrm{PGF}_{2} \alpha$.

\section{MATERIALE MÉTODOS}

No experimento foram utilizadas 387 vacas da raça Nelore (Bos indicus), pluríparas, com escore de condição corporal (ECC) entre 2,5 e 4 (escala de 1 a 5) e pósparto entre 45 a 60 dias, distribuídas em três réplicas similares, nas fazendas $A(n=197)$, $\mathrm{B}(\mathrm{n}=127)$ e $\mathrm{C}(\mathrm{n}=63)$ localizadas nos Estados do Maranhão e Pará, Brasil.

Os animais de cada réplica foram distribuídos homogeneamente em quatro tratamentos de acordo com período pósparto, escore de condição corporal, partida de sêmen e inseminador. As partidas de sêmen foram previamente aprovadas, seguindo os critérios recomendados pelo Colégio Brasileiro de Reprodução Animal (Henry e Neves, 1998).

Os protocolos experimentais com delineamento em fatorial estão ilustrados na figura 1 . No Dia (D0) do protocolo experimental, todos os animais receberam um dispositivo intravaginal de progesterona (1,9 g de P4, CIDR ${ }^{\circledR}$, Pfizer $)$ e foram distribuídos em dois tratamentos de acordo com o éster de estradiol utilizado: No tratamento $\mathrm{CE}$ receberam $1 \mathrm{mg}$ de Cipionato de Estradiol (CE) (0,5 ml de ECP ${ }^{\circledR}$, Pfizer). No tratamento BE foram aplicados $2 \mathrm{mg}$ de Benzoato de Estradiol(BE) (2ml de Estrogin ${ }^{\circledR}$, Farmavet). No Dia 7 (48 h antes da remoção do dispositivo) metade dos animais do tratamento $\mathrm{CE}$ e metade dos animais do tratamento BE foram contidos (sistema de quatro manejos) e receberam $10 \mathrm{mg}$ de Dinoprost(PGF2 $\alpha ; 5 \mathrm{mlde}$ Lutalyse ${ }^{\circledR}$, Pfizer). A outra metade dos animais de cada tratamento recebeu PGF $2 \alpha$ somente na ocasião da remoção do dispositivo intravaginal (Dia 9) (sistema de três manejos). Os animais foram alocados de acordo com os tratamentos, empregando-se delineamento fatorial 2x2 (Cipionato vs. Benzoato e PGF$D 7$ vs. $P G F-D 9)$ e compondo quatro grupos experimentais (CED7, $C E D 9$, $B E D 7 \mathrm{e} B E D 9$ ). Adicionalmente, ainda na ocasião da remoção do dispositivo (D9), independentemente do tratamento, todas as fêmeas receberam $1 \mathrm{mg}$ de Cipionato de Estradiol como indutor de ovulação e tiveram os bezerros removidos por 48 horas. No dia 11 , todas as fêmeas foram inseminadas em tempo fixo, num intervalo máximo de quatro horas (48 a 52 h após a retirada dos dispositivos).

O diagnóstico de gestação foi realizado por ultra-sonografia (CHISON 500VET, USProducts do Brasil, transdutor linear transretal de 5,0 MHz) aos 30 dias após a IATF.

Para a variável taxa de concepção foi adotada a distribuição binomial de Poisson (gestantes e não-gestantes; GUIDED DATA ANALYSIS do $\mathrm{SAS}^{\circledR}$; SAS, 2001). As médias dos quadrados mínimos foram geradas pelo PROC GENMOD do SAS ${ }^{\circledR}$ e comparadas por contrastes ortogonais. As variáveis classificatórias consideradas no modelo estatístico para verificação dos efeitos e interações foram: tratamento, réplica e animal. 


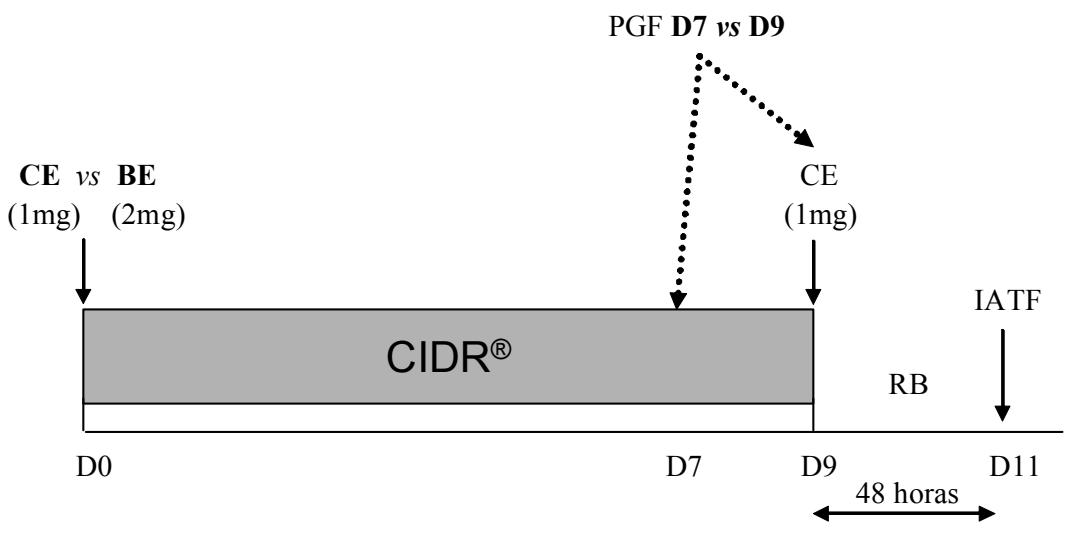

RB: Remoção de bezerros; CE: Cipionato de estradiol; BE: Benzoato de estradiol; PGF:

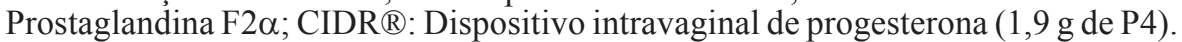

Figura 1. Experimental protocol. (Protocolo experimental).

\section{RESULTADOSEDISCUSSÃO}

A taxa de concepção total foi de $47,29 \%$ (183/387). Não houve interação entre réplicas e tratamentos, mas houve diferença significativa entre as três réplicas, sendo a primeira com 54,31\% (107/197) de concepção, a segunda com $42,53 \%(54 / 127)$ e a terceira e última réplica com $34,92 \%(22 / 63)$, fato ocorrido principalmente em resposta à interação réplica/ECC/inseminador.

A taxa de concepção foi similar ( $p>0,05)$ nos tratamentos experimentais (tabela I).

Tanto o cipionato quanto o benzoato de estradiol produziram similares taxas de concepção $(p>0,05)$. Comprovou-se que ambos são eficientes em induzir atresia e emergência de nova onda folicular ao início do protocolo de IATF. Isto se deve à similaridade desses ésteres de estradiol em inibirem a pulsatilidade de FSH e induzirem atresia folicular, embora o cipionato apresente meia-vida e clearance sanguíneo mais longos.

Considerou-se a hipótese de que o aumento da secreção pulsátil de LH durante o período entre a luteólise induzida pela PGF $2 \alpha$ e a remoção do progestágeno permitiria um crescimento mais expressivo do folículo ovulatório em animais com corpo lúteo em fase de diestro. Entretanto, nas

Tabela I. Efeito do éster de estradiol (BE ou $E C P)$ no DO e do momento da administração de $P G F_{2} \alpha$ (D7 ou D9) na taxa de concepção de vacas Nelore (Bos indicus) inseminadas em tempo fixo. (Effect of estradiol ester (BE or $E C P$ ) in D0 and moment of $\mathrm{PGF}_{2} \alpha$ administration (D7 or D9) on conception rate of fixed-time inseminated Nellore (Bos indicus) cows).

\begin{tabular}{lcc}
\hline D0 & PGF & Taxa de concepção \% \\
\hline BE & D7 & $45,3(43 / 95)$ \\
& D9 & $48,5(48 / 99)$ \\
CE & D7 & $52,1(50 / 96)$ \\
& D9 & $43,3(42 / 97)$ \\
Efeitos principais & & \\
BE & - & $46,9(91 / 194)$ \\
CE & - & $47,7(92 / 193)$ \\
- & D7 & $48,7(93 / 191)$ \\
- & D9 & $45,9(90 / 196)$ \\
\hline
\end{tabular}

Não houve diferença significativa entre tratamentos $(p>0,05)$.

BE: Benzoato de estradiol; CE: Cipionato de estradiol; PGF: Prostaglandina F2 $\alpha$; D7: dia sete do protocolo/48 horas antes da remoção do CIDR®; D9: dia nove do protocolo/remoção do CIDRß. 
condições experimentais aqui apresentadas, esta provável variação no crescimento folicular ocasionada pela administração de PGF2 $\alpha$ no $\mathrm{D} 7$ em relação ao D9 não influenciou significativamente a taxa de concepção em vacas Nelore (Bos indicus) ( $p>0,05$; tabela I). Vale ainda ressaltar que, em pesquisas anteriores, observou-se alto percentual de anestro em fêmeas zebuínas criadas extensivamente, sobretudo primíparas, antes dos 60 dias pós-parto, o que provavelmente não justifica o manejo adicional dos animais para a administração de PGF $2 \alpha 48$ horas antes da remoção do dispositivo intravaginal.

Estes resultados sugerem que é possível utilizar protocolo de IATF com três manejos, sendo a aplicação de PGF2 $\alpha$ no momento da retirada do dispositivo intravaginal de progesterona, diminuindo custos, mão-deobra e possível estresse dos animais.

Este estudo forneceu informações que

\section{BIBLIOGRAFIA}

Barros, C.M. e Ereno, R.L. 2004. Avanços em tratamentos hormonais para a inseminação artificial com tempo fixo (IATF) em bovinos de corte. Acta Scientiae Veterinariae, 32 (Supl): 23-34.

Baruselli, P.S., Reis, E.L., Marques, M.O., Nasser, L.F. and Bó, G.A. 2004. The use of hormonal treatments to improve reproductive performance of anestrous beef cattle in tropical climates. Anim. Reprod. Sci., 82-83: 479-486.

Bó, G.A., Adams, G.P., Nasser, L.F., Pierson, R.A. and Mapletoft, R.J. 1993. Effect of estradiol valerate on ovarian follicles, emergence of follicular waves and circulating gonadotropins in heifers. Theriogenology, 40: 225-239.

Bó, G.A., Adams, G.P., Caccia, M., Martínez, M.F., Pierson, R.A. and Mapletoft, R.J. 1995. Ovarian follicular wave emergence after treatment with progestogen and estradiol in cattle. Anim. podem direcionar o correto uso da IATF, na perspectiva de viabilizar a técnica em bovinos e auxiliar na elaboração de projetos futuros. Contudo, o nosso, dentre vários outros grupos de pesquisa, evidencia a necessidade de conduzir novas réplicas experimentais, considerando que diferentes condições de manejo animal influenciam grandemente nos resultados finais dos programas de sincronização da ovulação em bovinos.

\section{AGRADECIMENTOS}

Ao PIBIC-UFRA pela bolsa concedida, aos Laboratórios Pfizer do Brasil pelo fornecimento dos fármacos utilizados, à empresa USProducts do Brasil, pelo aparelho de ultra-som e aos proprietários e técnicos das fazendas Calumbi, Palmar e Maarambaia pelo apoio e disponibilização dos animais, sem os quais o presente trabalho não poderia ser realizado.

Reprod. Sci., 39: 193-204.

Henry, M. e Neves, J.P. 1998. Manual para exame andrológico e avaliação de sêmen animal. Colégio Brasileiro de Reprodução Animal. $2^{a}$ ed. Belo Horizonte. 49 pp.

Dieleman, S.J., Bevers, M.M., Van Tol, H.T.M. and Willemsw, A.H. 1986. Peripheral plasma concentrations of estradiol, progesterone, cortisol, LH and prolactin during the estrous cycle in the cow, with emphasis on the perioestrus period. Anim. Reprod. Sci., 10: 275-92.

Galina, C.S., Orihuela, A. and Bubio, I. 1996. Behavioural trends affecting oestrus detection in Zebu cattle. Anim. Reprod. Sci., 42: 465-470. SAS. 2001. Statistical analysis system. SAS user's guide: statisics. 8.0 version. SAS. Cary. 846 pp. Yavas, Y. and Walton, J.S. 2000. Induction of ovulation in postpartum suckled beef cows: A review. Theriogenology, 54: 1-25. 